Research Article

\title{
The Protective Effect of Cx43 Protein-Mediated Phosphocreatine on Myocardial Ischemia/Reperfusion Injury
}

\author{
Chen-xi Wang $\mathbb{D}^{1},{ }^{1}$ Jun-jun Guo, ${ }^{1}$ An-jie Di, ${ }^{1}$ Yu Zhu, ${ }^{1}$ Wei-min Han, ${ }^{1}$ An-ran Cheng, \\ Cheng Li, ${ }^{1}$ Rui-chan Si, ${ }^{1}$ Tian-shu Lan, ${ }^{2}$ Ran Zhang, ${ }^{1}$ Hong-li Liu $\mathbb{D}^{3},{ }^{3}$ and Guo-liang Yan $\mathbb{D}^{1}$ \\ ${ }^{1}$ School of Medicine, Xiamen University, Xiamen 361100, China \\ ${ }^{2}$ Key Laboratory of Functional and Clinical Translational Medicine, Fujian Province University, Xiamen Medical College, \\ Xiamen 361100, China \\ ${ }^{3}$ Department of Gynecology, Women and Children's Hospital, School of Medicine, Xiamen University, Xiamen 361100, China
}

Correspondence should be addressed to Hong-li Liu; doc79924763@163.com and Guo-liang Yan; zhuanyiyan@126.com

Received 18 September 2020; Revised 18 December 2020; Accepted 8 January 2021; Published 23 January 2021

Academic Editor: Paolo Severino

Copyright ( $) 2021$ Chen-xi Wang et al. This is an open access article distributed under the Creative Commons Attribution License, which permits unrestricted use, distribution, and reproduction in any medium, provided the original work is properly cited.

Objectives. To verify the protective effect of phosphocreatine on myocardium in an ischemic model and the possible mechanism of action. Methods. The model of myocardial ischemia/reperfusion (I/R) was established by the ligation balloon method. $30 \mathrm{SD}$ rats were randomly divided into three groups, $n=10$ in each group. Sham operation group: the coronary artery was not blocked and observed for 120 minutes. The ischemia/reperfusion $(I / R)$ group was given ischemia for 30 minutes and ischemia reperfusion for 90 minutes. Phosphocreatine (PCr) group: after 30 minutes of ischemia, the rats were intraperitoneally injected with $\mathrm{PCr}$ (200 mg/ $\mathrm{kg}$ ) for 90 minutes. The animal groups of myocardial ischemia/reperfusion model in vitro were the same as those in vivo. The heart was removed by thoracotomy and washed immediately in $\mathrm{H}-\mathrm{K}$ buffer solution. Then, the heart was installed on the Langendorff instrument. The concentration of PCr perfusion fluid in the PCr group was $10 \mathrm{mmol} / \mathrm{L}$. The changes in coronary blood flow in isolated myocardium were recorded. The heart rate and electrocardiogram were recorded by RM6240BT. At the end of the experiment, myocardial pathological sections and $\mathrm{Cx} 43$ immunofluorescence staining were made, and the contents of malondialdehyde (MDA) in myocardial tissue were detected. Results. Phosphocreatinine treatment improved the myocardial ischemia model, performance in electrocardiogram (ECG) changes (ST segment apparent), and histological changes (decrease in necrotic myocardial cells, inflammatory cell infiltration, and a reduction in myocardial edema). At the same time, MDA decreased, while coronary blood flow and $\mathrm{Cx} 43$ expression significantly improved. Conclusions. Phosphocreatine can improve the electrocardiogram and restore histologic changes in ischemic myocardium and coronary blood flow. The postulated mechanism is by inhibiting the generation of free oxygen radicals and restoring the expression of $\mathrm{Cx} 43$ protein.

\section{Introduction}

More people have died because of cardiovascular diseases (CVD) than any other diseases in the world [1]. Ischemic heart disease (IHD) is the most prevalent CVD and is defined clinically as acute myocardial infarction (AMI) or angina pectoris [2]. AMI afflicts human health resulting in millions of deaths worldwide. It is the main factor of chronic heart failure with a mortality rate of approximately $10 \%$ [3]. At present, the early opening of the culprit blood vessels is the most effective treatment method for acute myocardial injury. However, the resulting reperfusion injury cannot be averted because of its serious effects [4]. Myocardial ischemia/reperfusion ( $\mathrm{I} / \mathrm{R})$ injury is a complicated pathophysiological disease process that is associated with various factors and pathways [5-9]. A possible cause of damage induced by $\mathrm{I} / \mathrm{R}$ is the "reactive oxygen species" (ROS) produced during this process [10]. The overproduction of ROS, namely, hydroxyl radicals, hydrogen peroxide, and superoxide radicals is one of the $\mathrm{I} / \mathrm{R}$ complications which are able to cause severe damage to reproductive tissues. The elevated levels of ROS result in DNA damage, endothelial 
destruction, and apoptosis of granulosa cells $[11,12]$. Oxidative stress could be originated from the perturbation in the balance of prooxidant and antioxidant agents in which the human body would not be capable of eliminating the excessive amount of ROS from the body [13]. Multiple mechanisms participate in I/R-induced tissue damage, including the increased production of ROS, elevation of proinflammatory mediators, and the initiation of proapoptotic factors in different tissues.

Several studies have reported that testicular ischemia/ reperfusion incremented the oxidative stress and decreased the level of "antioxidant enzymes" $[10,14]$. The use of tadalafil, verapamil, and the combination of them can protect the testis tissue against IR injury [15].

There are multiple mechanisms that have been proposed to underlie the pathology, including oxidative damage $[16,17]$, energy metabolic disturbance, and calcium overload [18]. However, the deterioration in intracellular ATP generation is likely to result in repeated ischemia during reperfusion [19]. To lessen the myocardial I/R injury after cardiac arrest, it is important to reduce energy consumption while increasing the energy supply to the myocardium. Prevention and management of myocardial I/R injury is a key step in coronary heart disease surgery and is becoming a major clinical issue in the treatment of IHD.

Exogenous phosphocreatine (PCr), an ATP buffer, is a high-energy phosphate admixture and has been shown to play a protective role in I/R-injured myocardial tissue $[20,21]$. However, the mechanism by which it protects ischemia-injured myocardial tissue has not been fully elucidated. The aim of this paper was to explore the protective effects of PCr on myocardial infarction in rats and preliminarily discuss the mechanisms associated with oxidative stress and relevant protein levels.

\section{Materials and Methods}

2.1. Animals. 60 healthy male SD rats weighing 240-280 g were acquired from Slac Laboratory Animal Co. Ltd. (Shanghai, China). The laboratory animal license number is SCXK (FUJIAN)-2007-0001. The experiment obtained the necessary approval from the Animal Ethics Committee of Xiamen University. With a relative humidity of $55 \pm 5 \%$ in a pathogen-free facility, the animals were kept under standard laboratory conditions at $22 \pm 2{ }^{\circ} \mathrm{C}$. All procedures were in accordance with the Institutional Animal Care and Use Committee (IACUC) guidelines and the Laboratory animals guidelines for ethical review of welfare (GB/T 35892-2018).

2.2. Drugs and Instruments. Sodium creatine phosphate (PCr), number 922-32-7, was obtained from the Dalian Meilun Biotechnology Co., Ltd. MDA Kits, no. A003-2, were obtained from the Nanjing Jiancheng Bio Co. (Nanjing, China). Antibodies of connexin 43 (Cx43), no. C6219, were obtained from Sigma Bio Co. (MO63103, USA). KrebsHenseleit fluid ( $\mathrm{k}-\mathrm{h}$ fluid) was composed at a ratio of $120 \mathrm{mmol} / \mathrm{L} \mathrm{NaCl}, 1.2 \mathrm{mmol} / \mathrm{L} \mathrm{KH}_{2} \mathrm{PO}_{4}, 1.2 \mathrm{mmol} / \mathrm{L} \mathrm{CaCl}_{2}$, $1.2 \mathrm{mmol} / \mathrm{L} \quad \mathrm{MgSO}_{4}, \quad 25 \mathrm{mmol} / \mathrm{L}$ sodium acetate, and
$11 \mathrm{mmol} / \mathrm{L}$ glucose, $\mathrm{pH}$ 7.4. In the experiment, a mixture of $95 \% \mathrm{O}_{2}$ and $5 \% \mathrm{CO}_{2}$ was used. Ringer solution composition was $6.5 \mathrm{~g} \mathrm{NaCl}, \quad 0.12 \mathrm{~g} \mathrm{CaCl}_{2}, \quad 0.14 \mathrm{~g} \mathrm{KCl}, \quad 0.10 \mathrm{~g}$ $\mathrm{Na}_{2} \mathrm{HPO}_{4} \cdot 12 \mathrm{H}_{2} \mathrm{O}, 0.20 \mathrm{~g} \mathrm{NaHCO}$, and $2.00 \mathrm{~g}$ glucose, in $1.0 \mathrm{~L}$ distilled water. Other reagents employed were either domestic or imported analytical reagents.

Multichannel biological signal acquisition instrument (RM6240BT, Chengdu instrument factory, Chengdu, China) and multichannel biological signal acquisition and processing system (version 3.0) were used. A small animal ventilator, number HX-101E, (Chengdu Taimeng Software Co., Ltd., Chengdu, China), was used. A Heart Langendorff irrigation device (number LGF-18, Chengdu instrument factory, Chengdu, China) was used. A visible spectrophotometer (number V-1100D) and MAPADA were also used to assess absorbance.

\subsection{Model Preparation and Grouping}

\subsubsection{The Surgical Process of a Myocardial I/R Model.} The model of myocardial ischemia reperfusion was established by ligating the left anterior descending (LAD) coronary artery and releasing the ligation line [22, 23]. The blunt head guide wire of the rat's upper teeth was lifted up from the root of the tongue and inserted into the trachea along the direction of cricoid cartilage, and then the ventilator was connected. The rats were anesthetized by intraperitoneal injection of pentobarbital sodium solution ( $50 \mathrm{mg} / \mathrm{kg}$ ). The skin between the second and fifth ribs of the left chest was incised with a scalpel, and the subcutaneous tissue, serratus anterior, and pectoralis major muscle were bluntly separated. The chest expander was placed between 2 and 3 ribs to fully expose the heart. The LAD coronary artery was located, the ligation line was 6-0 operation line, the depth of the needle was $2 \mathrm{~mm}$, and the width was $2-3 \mathrm{~mm}$.

\subsubsection{Establishment of a Myocardial $I / R$ Model In Vivo.} $30 \mathrm{SD}$ rats were randomly divided into three groups, $n=10$ in each group. The control group (sham operation group): the coronary artery was not blocked and observed for 120 minutes. The ischemia/reperfusion (I/R) group was given ischemia for 30 minutes and ischemia reperfusion for 90 minutes. The creatine phosphate (PCr) group: after 30 minutes of ischemia, the rats were intraperitoneally injected with $200 \mathrm{mg} / \mathrm{kg} \mathrm{PCr}$ (the concentration of PCr was $40 \mathrm{mg} /$ $\mathrm{ml}$ ) and then given ischemia reperfusion for 90 minutes. The heart rate and electrocardiogram were recorded by RM6240BT. In the experiment, purple cyanosis and electrocardiogram II lead ST elevation in the anterior wall of the left ventricle developed into myocardial ischemia.

\subsubsection{Establishment of a Myocardial I/R Model In Vitro.} The animal groups of the myocardial I/R model in vitro were the same as those in vivo. The heart was removed by thoracotomy and washed immediately in $\mathrm{H}-\mathrm{K}$ buffer solution [23]. Then, the heart was installed on Langendorff instrument. The concentration of PCr perfusion fluid in PCr group 
was $10 \mathrm{mmol} / \mathrm{L}$. The changes in the coronary blood flow $(\mathrm{MCF})$ in isolated myocardium were recorded by collecting cardiac output over $1 \mathrm{~min} . \mathrm{MCF}=$ cardiac effluent per minute/total ventricular weight. The heart rate and electrocardiogram were recorded by RM6240BT.

\subsection{Sample Collection and Processing}

2.4.1. H\&E Immunoflorescence Staining. Specimens from different models were examined. All specimens were implanted in OCT and frozen according to routine histological methods [19]. Slices were stored at $-80^{\circ} \mathrm{C}$. The specimens from different models were then stained with hematoxylin and eosin (H\&E). Frozen tissue slices were removed from $-80^{\circ} \mathrm{C}$ and then incubated at $4^{\circ} \mathrm{C}$ for approximately 20 minutes. The sections were absorbed using 4\% paraformaldehyde for 15 minutes, washed twice in PBS washing solution for 5 minutes, and then rinsed in $0.2 \%$ Triton X-100 for 5 minutes. After blocking for 30 minutes with 5\% skimmed milk, the sections were then incubated at $4^{\circ} \mathrm{C}$ overnight in primary antibody: rabbit anti-rat $(1: 10000$; Sigma). The sections were then washed three times. With overnight incubation, the sections were incubated in the dark for 1 hour at $37^{\circ} \mathrm{C}$ with rat anti-rabbit FITC secondary antibody (1:300; Invitrogen, Carlsbad, CA). The sections were then sealed with mounting medium encasing and DAPI. The fluorescence microscope was used for observation.

2.4.2. MDA and Detection. For detection of MDA, manufacturer instructions were followed. Briefly, to assess the effect of $\mathrm{PCr}$ on oxidative stress, the MDA concentration $(\mathrm{nmol} / \mathrm{mg})$ was determined using the thiobarbital acid (TBA) method.

2.5. Statistics. All data are presented as the mean \pm standard deviation (SD). Electrocardiography parameters were compared using paired $t$-tests, while the remaining data were assessed by one-way analysis of variance (ANOVA). A $P$ value $<0.05$ was recognized to be statistically significant; $P<0.01$ and $P<0.001$ indicated highly significant differences. All analyses were performed using Graph Pad Prism $5{ }^{\circledR}$ (Graph Pad, Inc., La Jolla, CA, USA) software.

\section{Results}

\subsection{Electrocardiography Changes}

3.1.1. The Electrocardiogram Was Used to Monitor Changes in ECG Caused by Ischemic Reperfusion in Rats. Ischemia immediately induced a progressive increase in ST height and prolongation of the QT segment, and the amplitude of $\mathrm{P}, R$, and $T$ waves all increased. After ischemia, ST elevation and QT elongation were significantly different from those of the blank control $(P<0.001)$, demonstrating that the myocardial ischemia was obvious after the ligation of the left anterior descending branch. After $10 \mathrm{~min}$ of reperfusion, the ST segment height and the QT segment both declined, which proved that the reperfusion was successful. After PCr treatment, the ST segment decreased and the QT segment returned to normal levels. Compared with the control group, there was no statistically significant difference $(P>0.05)$ observed, indicating that PCr exerted a good therapeutic effect on myocardial ischemia. At the same time, in the PCr group, the changes in ST segment, ventricular tachycardia (VT), and ventricular fibrillation (VF) were significantly lower than those of the ischemia/reperfusion group. $\mathrm{P}, T$, and $R$ waves and the QT interval were significantly smaller than the ischemia/reperfusion group, while the heart rate decreased to a normal level. The results are shown in Figure 1 and Tables 1 and 2.

3.2. Pathological Staining of the Heart. The histologic changes induced immediately after 90 min of reperfusion were evaluated through hematoxylin and eosin staining. Compared to normal myocardial tissue (d, g), myocardial changes were noted immediately after $30 \mathrm{~min}$ ischemia. These included coagulation and necrosis, cardiomyocyte disorganization, stromal edema $(\star)$, hemorrhage, and increased infiltration of inflammatory cells $(\triangleleft)(e, h)$. With the application of PCr, cardiomyocyte necrosis decreased, inflammatory cell infiltration decreased, and there was a relief of myocardial edema (e, i). These data indicated that PCr was able to improve the pathologic damage inflicted upon heart tissue due to myocardial ischemia. The results are shown in Figure 2.

3.3. Changes in Coronary Blood Flow in Isolated Rat Hearts. In order to indirectly estimate the contractile capacity of cardiomyocytes after $30 \mathrm{~min}$ ischemia, coronary blood flow was assessed once every five minutes using a cylinder to collect blood at the end of the reperfusion period. As shown in Figure 3, the I/R group resulted in a decrease in coronary blood flow compared with the control group, while the PCr group resulted in a significantly increased coronary blood flow compared with the/ $\mathrm{R}$ group. However, this did not return to the control group level.

3.4. MDA Content after Ischemia Reperfusion and $\mathrm{PCr}$ Treatment. As shown in Figure 4, compared with the control group, I/R resulted in higher accumulation of lipid peroxides $(P<0.001)$, while the MDA was significantly decreased compared with the I/R group $(P<0.001)$ but did not return to control levels.

3.5. Immunoflorescence Staining of Cx43 in Rat Hearts. To analyze the distribution of $\mathrm{Cx} 43$, heart tissue was labeled with $\mathrm{Cx} 43$ antibody and DAPI. In the control group, the nucleus was clearly stained with DAPI, and Cx43 was strongly expressed in the cell membrane (Figures 5(a)-5(c) and Figures $6(\mathrm{a})-6(\mathrm{c}))$. In contrast, in the I/R group, DAPI staining also showed the nucleus, but the expression of $\mathrm{Cx} 43$ in the cell membrane was significantly reduced (Figures 5(d)-5(f) and Figures 6(d)-6(f)). In the PCR group, the recovery of $\mathrm{Cx} 43$ expression in the cell membrane was 

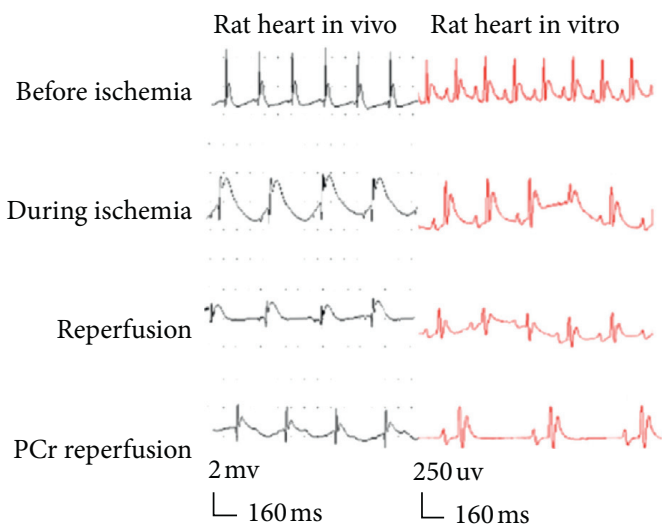

FIGURE 1: Representative composite electrocardiography changes of rats in vivo and in vitro before ischemia, during ischemia, reperfusion, and PCr reperfusion.

TABLE 1: Electrocardiographic parameters as calculated from the composite recordings in rats in vivo $(n=10)$.

\begin{tabular}{lcccccc}
\hline Group & Heart rate $(\mathrm{BMP})$ & ST height $(\mathrm{mV})$ & QT $(\mathrm{ms})$ & P amplitude $(\mathrm{mV})$ & $\mathrm{R}$ amplitude $(\mathrm{mV})$ & T amplitude $(\mathrm{mV})$ \\
\hline Control & $356.1 \pm 40.2$ & $14.5 \pm 10.7$ & $76.7 \pm 14.1$ & $2.77 \pm 1.53$ & $6.04 \pm 1.55$ & $2.03 \pm 1.02$ \\
I/R & $236.5 \pm 42.1^{* *}$ & $200.7 \pm 33.2^{* * *}$ & $105.4 \pm 17.3^{*}$ & $5.84 \pm 2.58^{*}$ & $8.82 \pm 2.01^{*}$ & $3.72 \pm 1.17^{*}$ \\
PCr & $313.1 \pm 37.2^{\#}$ & $15.3 \pm 12.6^{\# \#}$ & $80.2 \pm 14.3^{\#}$ & $3.04 \pm 1.65^{\#}$ & $6.22 \pm 1.23^{\#}$ & $2.08 \pm 1.04^{\#}$ \\
\hline
\end{tabular}

Note: ${ }^{*} P<0.05,{ }^{* *}<0.01,{ }^{* * *}<0.001$ versus the control group. ${ }^{\#} P<0.05,{ }^{\# \#} P<0.01,{ }^{\# \# *} P<0.001$ versus the I/R group. PCr group measurements were not all statistically significant, compared with the control group $(P>0.05)$.

TABLE 2: Electrocardiographic parameters as calculated from the composite recordings in rats in vitro $(n=10)$.

\begin{tabular}{lcccccc}
\hline Group & Heart rate $(\mathrm{BMP})$ & ST height $(\mathrm{mV})$ & QT $(\mathrm{ms})$ & P amplitude $(\mathrm{mV})$ & $\mathrm{R}$ amplitude $(\mathrm{mV})$ & T amplitude $(\mathrm{mV})$ \\
\hline Control & $267.3 \pm 32.4$ & $20.5 \pm 10.4$ & $82.1 \pm 21.3$ & $2.61 \pm 1.37$ & $6.11 \pm 1.74$ & $1.98 \pm 1.09$ \\
I/R & $166.6 \pm 30.8^{* *}$ & $280.7 \pm 43.5^{* * *}$ & $144.0 \pm 35.4^{*}$ & $5.77 \pm 2.49^{*}$ & $8.95 \pm 2.17^{*}$ & $3.87 \pm 1.23^{*}$ \\
PCr & $254.7 \pm 25.2^{\# \#}$ & $25.3 \pm 22.6^{\# \# \#}$ & $97.3 \pm 19.8^{\#}$ & $2.95 \pm 1.54^{\#}$ & $6.25 \pm 1.35^{\#}$ & $2.18 \pm 1.03^{\#}$ \\
\hline
\end{tabular}

Note: ${ }^{*} P<0.05,{ }^{* *} P<0.01,{ }^{* * *}<0.001$ versus control group. ${ }^{\#} P<0.05,{ }^{\#} P<0.01,{ }^{\# \#} P<0.001$ versus I/R group. PCr group measurements were not all statistically significant, compared with the control group $(P>0.05)$.

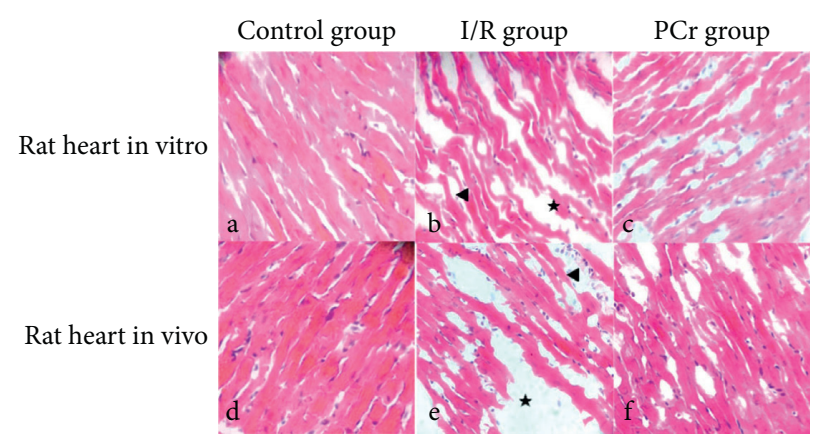

Figure 2: Histopathology of the hearts of rats. Hematoxylin and eosin staining; magnification of 40x ( $(\mathrm{a}-\mathrm{c})$ rat heart in vitro; (d-f) rat heart in vivo; (a, d), control group; (b, e) I/R group; (c, f) PCr group).

more or less the same as that of the blank control group (Figures 5(g)-5(i) and Figures 6(g)-6(i)). At the same time, the fluorescence intensity was analyzed. Compared with the control group, the fluorescence intensity of the $I / R$ group was significantly decreased, and the fluorescence intensity increased after PCr treatment. The results are shown in Table 3.

\section{Discussion}

Ischemic heart disease (IHD) still represents a large burden on individuals and health care resources worldwide. With the recent advances in cardiovascular research, IHD continues to be associated with both high morbidity and mortality globally [24]. IHD pathophysiology is more complex and multifaceted 


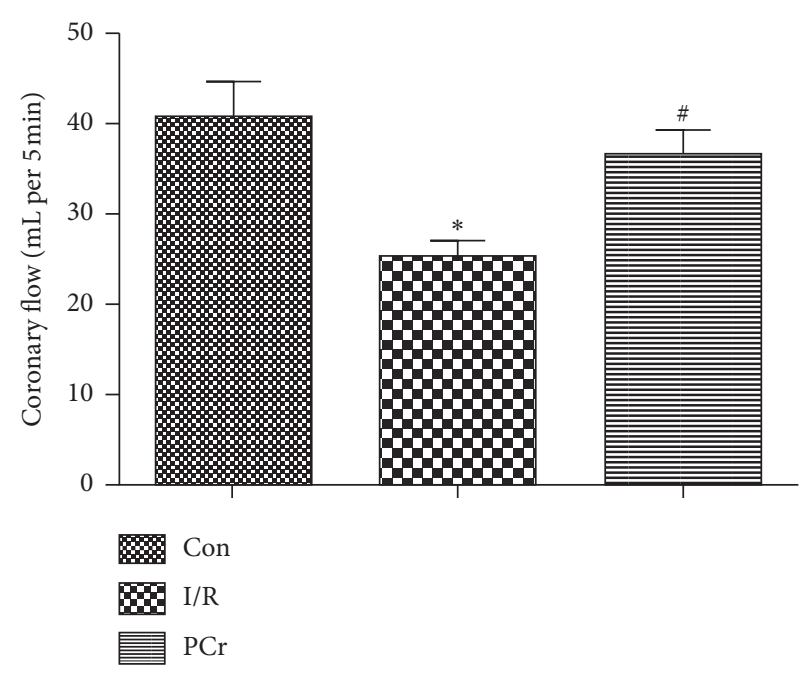

FIgURE 3: Changes in coronary flow in isolated rat hearts. Compared with the control group, the values in the I/R group had a significantly decreasing trend $\left(n=10,{ }^{*} P<0.05\right)$. Meanwhile, the values in the $\mathrm{PCr}$ group had a significantly increasing trend compared with the I/R group $\left(n=10,{ }^{\#} P<0.05\right)$.

than a single, simplistic, cause-effect event. A large percentage of patients with IHD have minimal or no epicardial coronary vascular disease. Microvascular disease plays an important role in the etiology of IHD, by regulating blood flow and oxygen and energetic substrates delivery, in the microcirculation-myocardium interaction [25]. In the last decades, coronary microcirculation function and structure abnormalities have been described as a relevant IHD pathogenic mechanism. Coronary microvascular dysfunction (CMD) represents a common pathophysiological mechanism of type II myocardial infarction. CMD may have consequences also on hemorheological features of blood flow in epicardial arteries, worsening coronary artery disease (CAD) and establishing a vicious circle, which contributes to myocardial ischemia $[26,27]$. CMD determines an inability of the coronary circulation to satisfy myocardial metabolic demands, due to the imbalance of coronary blood flow regulatory mechanisms, including ion channels, leading to the development of hypoxia, fibrosis, and tissue death, which may determine a loss of myocardial function, even beyond the presence of atherosclerotic epicardial plaques. For this reason, ion channels may represent the link among coronary microvascular dysfunction, ischemic heart disease, and consequent heart failure [26]. Myocardial ischemia is directly dependent on an impairment of the cross talk between myocardial energy state and coronary blood flow. The coronary macrovascular and microvascular disease may represent just a portion of the multifaceted pathophysiology of myocardial ischemia [25].

This coupled comorbidity of pathological ischemia and therapeutic reinjury of infarcted myocardium, namely, myocardial ischemia/reperfusion injury (MIRI), is particularly refractory to treatment $[28,29]$. Traditionally, MIRI can be due to reactive oxygen and nitrogen species (ROS/RNS) generation, reduced availability of nitric oxide (NO), $\mathrm{Ca}^{2+}$ overload, and mitochondrial permeability transition pore (mPTP) opening. From the regulation of the inflammatoryimmune response and the energy and metabolism in mitochondria to the epigenetic modification of chromatin, more and more novel molecular targets for MIRI and cardioprotection are being identified [30].

Furthermore, reperfusion therapy promotes the rapid recovery of blood flow to the myocardial ischemic zone but can result in further complications including diminished cardiac contractile and electrical conductivity function, and irreversible tissue necrosis [31]. Therefore, reducing reperfusion injury has become an important target of clinical research.

As a crucial energy substrate, $\mathrm{PCr}$ has a dual function of both storing and transporting ATP in energy metabolism. Exogenous PCr adds energy directly to the cell through the $\mathrm{PCr} / \mathrm{CK}$ system [32]. The pharmacokinetics of PCr displays a biphasic distribution in the blood, where the clearance rate of PCr is initially rapid after injection of a single dose. It is bound strongly to the heart due to its high affinity to the myocardium. Correspondingly, through this direct interaction, exogenous $\mathrm{PCr}$ may perform greatly in ischemic myocardium. It has been reported that, during periods of myocardial ischemia in rats, PCr conditioning could advance the levels of intracellular ATP and attenuate metabolic stress [33].

In order to check the protective effect of $\mathrm{PCr}$ on the myocardium of ischemic reperfusion injury, models of acute ischemia/reperfusion injury in rats were established to certify ECG and histopathological changes. There is evidence available to demonstrate the efficiency of ECG changes in the diagnosis of acute myocardial ischemia/reperfusion injury [34]. In our experiment, after PCr treatment, the ST segment declined and the QT segment returned to normal levels, demonstrating that $\mathrm{PCr}$ exerted a good therapeutic effect on myocardial ischemia. At the same time, in the PCr group, the changes in ST segment, ventricular tachycardia (VT), and ventricular fibrillation (VF) were significantly lower than those of the ischemia/reperfusion group. $\mathrm{P}, \mathrm{T}$, and $R$ waves and the QT interval were significantly smaller than in the ischemia/reperfusion group, while the heart rate dropped to normal levels.

In addition to $\mathrm{I} / \mathrm{R}$, the inflammatory response can exacerbate myocardial injury $[35,36]$. Myocardial infarction causes neutrophil infiltration into the ischemic area, and this infiltration can result in myocardial damage [37]. The histological changes to the myocardium after ischemia reperfusion were observed, and myocardial necrosis, tissue edema, and inflammatory cell infiltration were all apparent after myocardial ischemia. With $\mathrm{PCr}$ treatment, there were significant morphological differences observed. After PCr treatment, histopathology demonstrated that the myocardial injury could be reversed. Our experimental data are consistent with these 


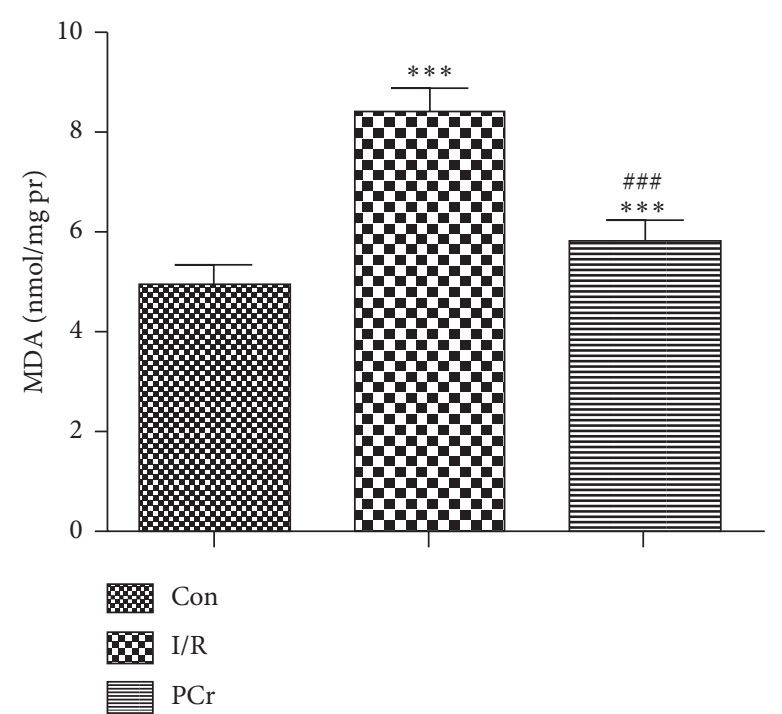

(a)

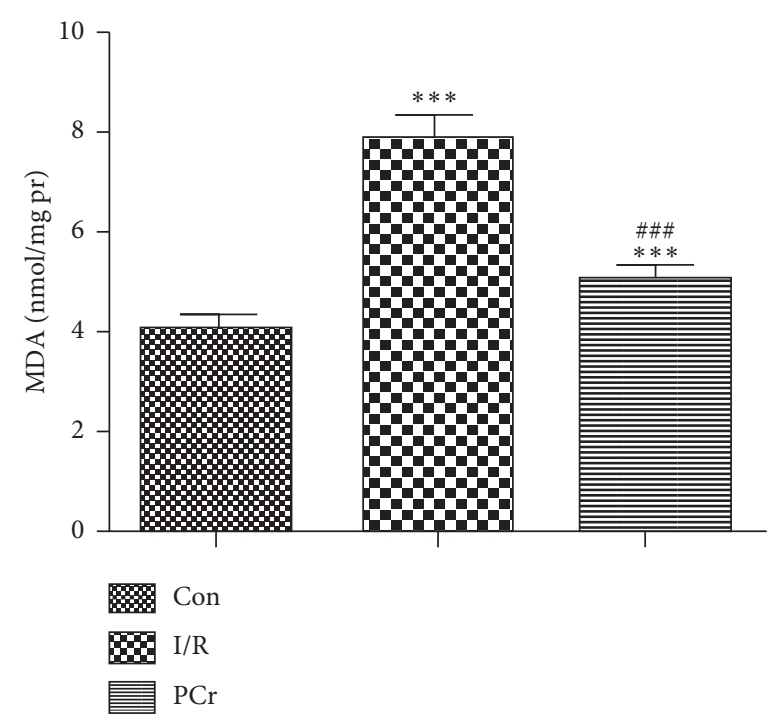

(b)

Figure 4: Changes in MDA content. (a) Rat heart in vitro and (b) rat heart in vivo. Compared with the control group, the I/R and PCr groups were significantly different $\left(n=10,{ }^{* * *} P<0.001\right)$; the $\mathrm{PCr}$ group shows a significant difference from the I/R group $(n=10$, \#\#\# $P<0.001)$.

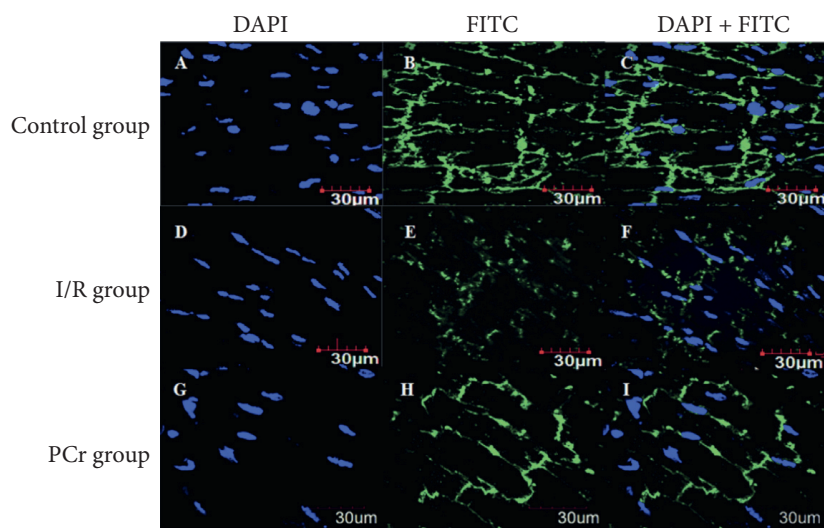

Figure 5: Immunoflorescence staining of Cx43 in rat hearts in vivo (200x). ((a) (b), (c)) Cx43 is mainly located at the intercalated discs of the control group. ((d) (e), (f)) In the I/R group, the Cx43 signal at the intercalated discs is lost and arranged irregularly. ((g) (h), (i)) PCr leads to a partial preservation of $\mathrm{Cx} 43$ at the intercalated discs after $\mathrm{I} / \mathrm{R}$.

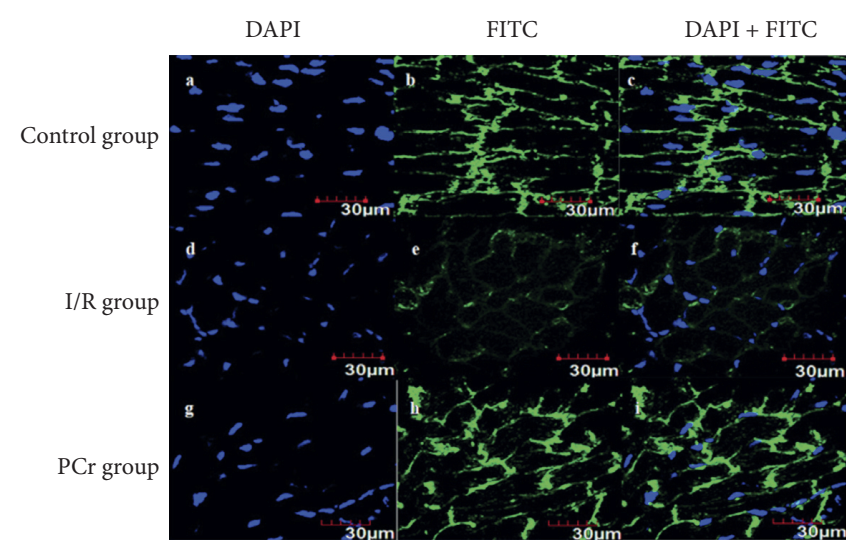

Figure 6: Immunoflorescence staining of Cx43 in rat hearts in vivo (200x). ((a) (b), (c)) Cx43 is mainly located at the intercalated discs of the control group. ((d) (e), (f)) In the I/R group, the Cx43 signal at the intercalated discs is lost and arranged irregularly. ((g) (h), (i)) PCr leads to a partial preservation of $\mathrm{Cx} 43$ at the intercalated discs after I/R. 
TABLE 3: The results of fluorescence intensity analysis.

\begin{tabular}{lcccccc}
\hline No. & $B$ & $E$ & $H$ & $b$ & $e$ & $h$ \\
\hline Constituency area & 1.85 & 1.63 & 1.85 & 1.94 & 1.94 & 1.94 \\
$\begin{array}{l}\text { Average fluorescence } \\
\text { intensity }\end{array}$ & 14.28 & 4.46 & 8.63 & 14.85 & 4.16 & 13.88 \\
\hline
\end{tabular}

phenomena. With the application of PCr, necrotic myocardial cells decreased, inflammatory cell infiltration diminished, and myocardial interstitial edema decreased. These data suggest that PCr could improve pathologic damage to heart tissue, which was precipitated by myocardial ischemia.

Acute ischemia reperfusion myocardial injury may include mechanisms associated with mitochondrial damage and consequent energy metabolic disorders, calcium overload, excitatory amino acid (EAAs) nerve toxicity, the accumulation of oxygen free radicals, and other inflammatory reactions. Among these mechanisms, oxidative stress injury is an important mechanism, which is also a currently researched activity. In the human body, the level of ROS and antioxidant compounds are in equilibrium as the overproduction of ROS results in oxidative stress. Oxidative stress could be originated from the perturbation in the balance of prooxidant and antioxidant agents in which the human body would not be capable of eliminating the excessive amount of ROS from the body [13]. Used to evaluate free radical-mediated myocardial cell injury, MDA is a crucial product of lipid peroxidation [38]. Our study confirmed that PCr inhibits the excessive generation of ROS and plays a protective role in cell damage by inhibiting MDA. In addition, PCr can increase blood flow to the coronary arteries after ische$\mathrm{mia} /$ reperfusion injury. These data show that PCr is effective at protecting and aiding the recovery of ischemia reperfusion in the heart.

Recently, the protective mechanism of PCr for myocardial ischemia reperfusion has been extensively researched. As the main connecting protein of cardiomyocytes, $\mathrm{Cx} 43$ provides a structural basis for the maintenance of the electrical physiological pulsation of the cardiomyocyte $[39,40]$. To explore whether the protective effect of $\mathrm{PCr}$ on myocardium after ischemic reperfusion injury was related to the expression of $\mathrm{Cx} 43$, we established a myocardial model of rat ischemia/ reperfusion injury and immunoflorescence staining to observe changes in $\mathrm{Cx} 43$. Our data showed that, in the control group, there was visible staining in the nucleus, Cx43 was expressed on the membrane at higher levels, while, in the I/R group, the expression of $\mathrm{Cx} 43$ abundance decreases, which was consistent with overall decreased cardiac function after myocardial ischemia. When the $\mathrm{PCr}$ treatment was initiated, the expression of $\mathrm{Cx} 43$ protein increased again, and levels approximated that of the control group. This is consistent with the overall recovery of cardiac function after PCr. The aforementioned data imply that the protective mechanism of $\mathrm{PCr}$ against cardiac ischemia/reperfusion injury may be directly or indirectly related to the increase and decrease in Cx43 protein expression.

For the first time, this study found that PCr may exert its effect on the myocardium by affecting the expression of $\mathrm{Cx} 43$. However, the exact mechanism of action needs further elucidation through future research.

\section{Conclusion}

Our study showed that PCr could be used to treat and improve myocardial ischemia in a model of ischemia/ reperfusion injury. The ST segment in the ECG and necrotic myocardial cells decreased, inflammatory cell infiltration decreased, myocardial interstitial edema decreased, and coronary blood flow was restored. One possible mechanism is through inhibition of the generation of oxygen free radicals and to protect the expression of $\mathrm{Cx} 43$ protein, thus restoring normal function to the myocardium.

\section{Data Availability}

The data used to support the findings of this study are available from the corresponding author upon request.

\section{Conflicts of Interest}

The authors declare no conflicts of interest in this work.

\section{Acknowledgments}

The authors thank Jingru Huang for her help with the laser scanning confocal microscope. This study was supported by the College Students' Innovative Entrepreneurial Training Plan Program (2020X0736), Education and Research Project for Young and Middle-aged Teachers of Fujian Province Education Department (JAT170714), and the Horizontal Subject (XDHT2020457A).

\section{References}

[1] I. Tzoulaki, P. Elliott, V. Kontis, and M. Ezzati, "Worldwide exposures to cardiovascular risk factors and associated health effects," Circulation, vol. 133, no. 23, pp. 2314-2333, 2016.

[2] V. Vaccarino, L. Badimon, R. Corti et al., "Ischaemic heart disease in women: are there sex differences in pathophysiology and risk factors?: position Paper from the Working Group on Coronary Pathophysiology and Microcirculation of the European Society of Cardiology," Cardiovascular Research, vol. 90, no. 1, pp. 9-17, 2011.

[3] M.-G. Perrelli, P. Pagliaro, and C. Penna, "Ischemia/reperfusion injury and cardioprotective mechanisms: role of mitochondria and reactive oxygen species," World Journal of Cardiology, vol. 3, no. 6, pp. 186-200, 2011.

[4] D. J. Hausenloy and D. M. Yellon, "Ischaemic conditioning and reperfusion injury," Nature Reviews Cardiology, vol. 13, no. 4, pp. 193-209, 2016. 
[5] K. Chen, W. Xie, B. Y. Luo et al., "Intestinal mucosal barrier is injured by bmp2/4 via activation of nf-kappa $\mathrm{b}$ signals after ischemic reperfusion," Mediators of Inflammation, vol. 2014, Article ID 901530, 11 pages, 2014.

[6] X. Li, J. Yang, J. Yang et al., "Rp105 protects against myocardial ischemia-reperfusion injury via suppressing tlr4 signaling pathways in rat model," Experimental and Molecular Pathology, vol. 100, no. 2, pp. 281-286, 2016.

[7] L. Ma, H. Liu, Z Xie et al., "Ginsenoside rb3 protects cardiomyocytes against ischemia-reperfusion injury via the inhibition of jnk-mediated nf-kappab pathway: a mouse cardiomyocyte model," PloS One, vol. 9, Article ID e103628, 2014.

[8] Y. K. Wang, F. Deng, J. Miao, H. Xie, and J. C. Feng, "Neuroprotection by carbenoxolone against ischemia injury involves pi3k/akt pathway," Clinical Laboratory, vol. 61, pp. 1561-1568, 2015

[9] T. Zhu, Q. Yao, X. Hu, C. Chen, H. Yao, and J. Chao, "The role of MCPIP1 in ischemia/reperfusion injury-induced HUVEC migration and apoptosis," Cellular Physiology and Biochemistry, vol. 37, no. 2, pp. 577-591, 2015.

[10] E. Yuluğ, S. Türedi, E. Karagüzel, Ö Kutlu, A. Menteşe, and A. Alver, "The short term effects of resveratrol on ischemia-reperfusion injury in rat testis," Journal of Pediatric Surgery, vol. 49, no. 3, pp. 484-489, 2014.

[11] M. Sapmaz-Metin, Y. Topcu-Tarladacalisir, Y. H. Uz et al., "Vitamin E modulates apoptosis and c-jun N-terminal kinase activation in ovarian torsion-detorsion injury," Experimental and Molecular Pathology, vol. 95, no. 2, pp. 213-219, 2013.

[12] H. Gharamaleki, K. Parivar, J. S. Rad, L. Roushangar, and M. Shariati, "Effects of extremely low-frequency electromagnetic field exposure during the prenatal period on biomarkers of oxidative stress and pathology of ovarian tissue in F1 generation," International Journal of Current Research and Review, vol. 5, no. 21, pp. 23-27, 2013.

[13] S. Majid, S. Malihe, S-H Abtahi-Eivary, N. Vahid, J. R. Poor Mohammad et al., "Effect of hydro-alcoholic extract of Olea europaea on apoptosis-related genes and oxidative stress in a rat model of torsion/detorsion-induced ovarian damage," Asian Pacific Journal of Reproduction, vol. 8, no. 4, pp. 148156, 2019.

[14] M. Ayan, U. Tas, E. Sogut et al., "Protective effect of thymoquinone against testicular torsion induced oxidative injury," Andrologia, vol. 48, no. 2, pp. 143-151, 2016.

[15] M. Ameli, M. Sadat Hashemi, M. Moghimian, and M. Shokoohi, "Protective effect of tadalafil and verapamil on testicular function and oxidative stress after torsion/detorsion in adult male rat," Andrologia, vol. 50, Article ID e13068, 2018.

[16] G. Ambrosio, J. L. Zweier, C. Duilio et al., "Evidence that mitochondrial respiration is a source of potentially toxic oxygen free radicals in intact rabbit hearts subjected to ischemia and reflow," Journal of Biological Chemistry, vol. 268, no. 25 , pp. 18532-18541, 1993.

[17] J. M. Brown, L. S. Terada, M. A. Grosso et al., "Hydrogen peroxide mediates reperfusion injury in the isolated rat heart," Molecular and Cellular Biochemistry, vol. 84, no. 2, pp. 173175, 1988.

[18] E. R. Vasques, J. E. Cunha, A. M. Coelho et al., "Trisulfate disaccharide decreases calcium overload and protects liver injury secondary to liver ischemia/reperfusion," PloS One, vol. 11, Article ID e0149630, 2016.

[19] A. Moriguchi, H. Otani, K. Yoshioka et al., "Inhibition of contractile activity during postconditioning enhances cardioprotection by restoring sarcolemmal dystrophin through phosphatidylinositol 3-kinase," Circulation Journal, vol. 74, no. 11, pp. 2393-2402, 2010.

[20] G. Landoni, A. Zangrillo, V. V. Lomivorotov et al., "Cardiac protection with phosphocreatine: a meta-analysis," Interactive CardioVascular and Thoracic Surgery, vol. 23, no. 4, pp. 637-646, 2016.

[21] L. H. Tang, Z. Y. Xia, B. Zhao, X. D. Wei, T. Luo, and Q. T. Meng, "Phosphocreatine preconditioning attenuates apoptosis in ischemia-reperfusion injury of rat brain," Journal of Biomedicine and Biotechnology, vol. 2011, Article ID 107091, 2011.

[22] L. I. Yan-ming, J. Liu, C.-c. Zhang, M. A. Xiao-hua, and J.-b. Gong, "Establishment of a modified myocardial ischemia reperfusion model in the SD rat," Journal of Medical Postgraduates, vol. 30, no. 4, pp. 413-416, 2017.

[23] X. M. Zhao, S. Sun, and X. H. Liu, "Experinmental model of myocardia ischemia/reperfusion in rat established by ligating coronary artery with capsule," China Microcirculation, vol. 11, pp. 206-207, 2007.

[24] Y.-w. Yao, G.-h. Zhang, Y.-y. Zhang et al., "Lipopolysaccharide pretreatment protects against ischemia/reperfusion injury via increase of HSP70 and inhibition of NF- $\kappa \mathrm{B}$," Cell Stress and Chaperones, vol. 16, no. 3, pp. 287-296, 2011.

[25] P. Severino, A. D'Amato, M. Pucci et al., "Ischemic heart disease pathophysiology paradigms overview: from plaque activation to microvascular dysfunction," International Journal of Molecular Sciences, vol. 21, no. 21, p. 8118, 2020.

[26] P. Severino, A. D’Amato, M. Pucci et al., "Ischemic heart disease and heart failure: role of coronary ion channels," International Journal of Molecular Sciences, vol. 21, no. 9, p. $3167,2020$.

[27] G. Heusch, A. Skyschally, and P. Kleinbongard, "Coronary microembolization and microvascular dysfunction," International Journal of Cardiology, vol. 258, pp. 17-23, 2018.

[28] D. M. Yellon and D. J. Hausenloy, "Myocardial reperfusion injury," New England Journal of Medicine, vol. 357, no. 11, pp. 1121-1135, 2007.

[29] D. J. Hausenloy and D. M. Yellon, "Myocardial ischemiareperfusion injury: a neglected therapeutic target," Journal of Clinical Investigation, vol. 123, no. 1, pp. 92-100, 2013.

[30] N.-B. Liu, M. Wu, C. Chen et al., "Novel molecular targets participating in myocardial ischemia-reperfusion injury and cardioprotection," Cardiology Research and Practice, vol. 2019, Article ID 6935147, , 2019.

[31] A. T. Turer and J. A. Hill, "Pathogenesis of myocardial ischemia-reperfusion injury and rationale for therapy," The American Journal of Cardiology, vol. 106, pp. 360-368, 2011.

[32] W. Zhang, H. Zhang, and Y. Xing, "Protective effects of phosphocreatine administered post-treatment combined with ischemic post-conditioning on rat hearts with myocardial ischemia/reperfusion injury," Journal of Clinical Medicine Research, vol. 7, no. 4, pp. 242-247, 2015.

[33] G. Prabhakar, L. Vona-Davis, D. Murray, P. Lakhani, and G. Murray, "Phosphocreatine restores high-energy phosphates in ischemic myocardium: implication for off-pump cardiac revascularization," Journal of the American College of Surgeons, vol. 197, no. 5, pp. 786-791, 2003.

[34] M. B. Preda and A. Burlacu, "Electrocardiography as a tool for validating myocardial ischemia-reperfusion procedures in mice," Comparative Med, vol. 60, pp. 443-447, 2010.

[35] L. Pourafkari, O. Visnjevac, S. Ghaffari, and N. Nader, "Statin drugs mitigate cellular inflammatory response after st elevation myocardial infarction, but do not affect in-hospital 
mortality," Journal of Cardiovascular and Thoracic Research, vol. 8, no. 1, pp. 34-39, 2016.

[36] Y. Tan, Y. Tu, D. Tian, C. Li, J.-K. Zhong, and Z.-G. Guo, "STelevation myocardial infarction following systemic inflammatory response syndrome: case report," Cardiovascular Journal of Africa, vol. 26, no. 3, pp. e1-e3, 2015.

[37] I.-W. Shin, I.-S. Jang, S.-M. Lee et al., "Myocardial protective effect by ulinastatin via an anti-inflammatory response after regional ischemia/reperfusion injury in an in vivo rat heart model," Korean Journal of Anesthesiology, vol. 61, no. 6, pp. 499-505, 2011.

[38] K. A. Roebuck, "Oxidant stress regulation of il-8 and icam-1 gene expression: differential activation and binding of the transcription factors ap-1 and nf-kappab," International Journal of Molecular Medicine, vol. 4, pp. 223-230, 1999.

[39] D. Alexander and G. Goldberg, "Transfer of biologically important molecules between cells through gap junction channels," Current Medicinal Chemistry, vol. 10, no. 19, pp. 2045-2058, 2003.

[40] T. Brandenburger, R. Huhn, A. Galas, B. H. Pannen, V. Keitel et al., "Remote ischemic preconditioning preserves connexin 43 phosphorylation in the rat heart in vivo," Journal of Translational Medicine, vol. 12, p. 228, 2014. 